\title{
A simple method for safe determination of the activity of palladium on activated carbon catalysts in the hydrogenation of cinnamic acid
}

\section{to hydrocinnamic acid}

Sajjad Mousavi ${ }^{1}$, Behzad Nazari ${ }^{1, *}$, Mohammad H. Keshavarz ${ }^{1, *}$, and Abdol-Khalegh Bordbar ${ }^{2}$

${ }^{1}$ Department of Chemistry, Malek-Ashtar University of Technology, Shahin-Shahr, 83145/115, Iran

${ }^{2}$ Department of Chemistry, University of Isfahan, Isfahan, 81746-73441, Iran

*To whom correspondence should be addressed; Tel:(0098)-0314-522-5071; Fax: (0098)-0314-522-5068; 1)

Professor Dr. M. H. Keshavarz, E-mail: mhkeshavarz@mut-es.ac.ir; keshavarz7@gmail.com; 2) Dr. B. Nazari,

E-mail: b.nazari49@gmail.com 


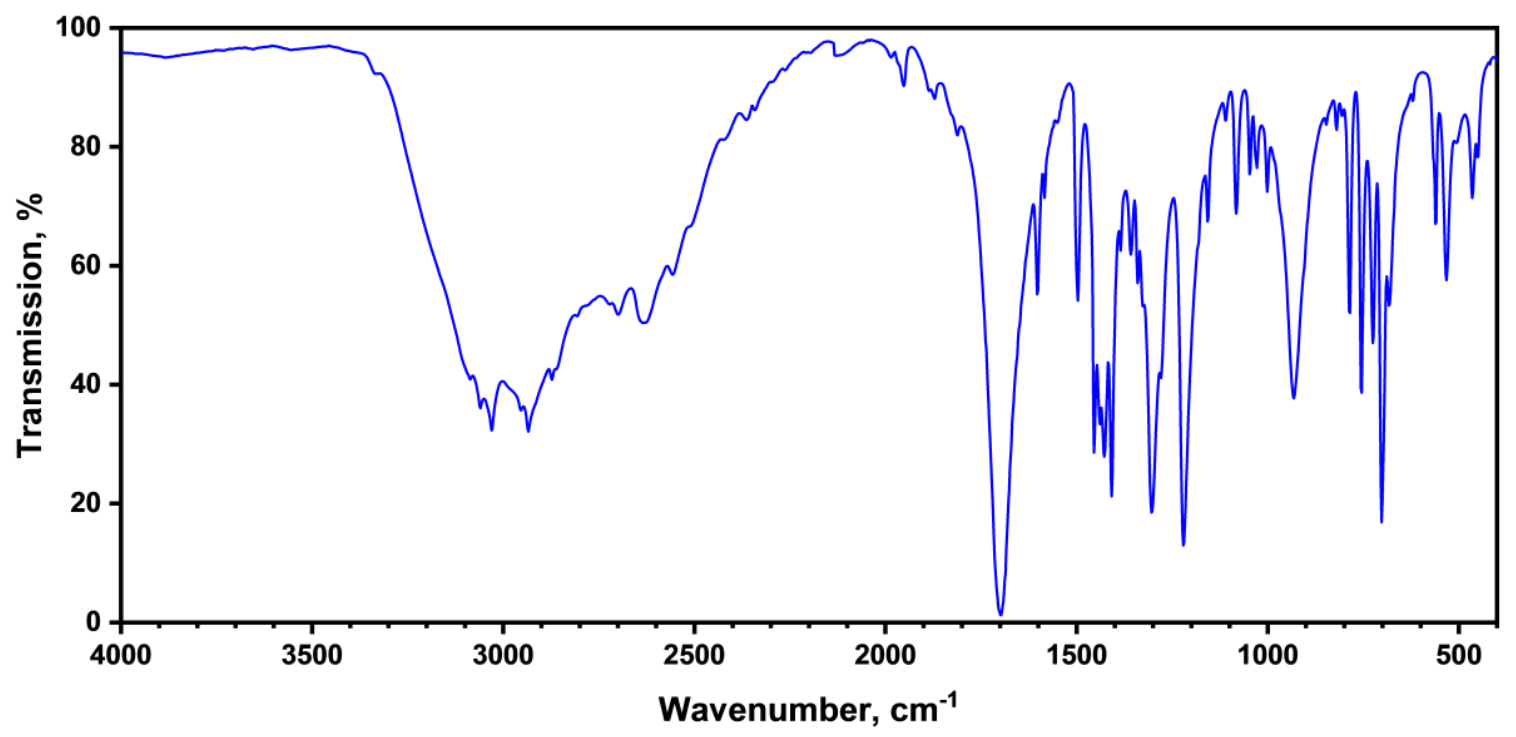

Figure S1. The FT-IR spectrum of the hydrocinnamic acid (HCA) synthesized by catalytic transfer hydrogenation (CTH) reaction

e d $f$

${ }^{1} \mathrm{H}-\mathrm{NMR}$

(400 MHz, [D6] DMSO, TMS)<smiles>CC(C)(C)Cc1ccccc1</smiles>

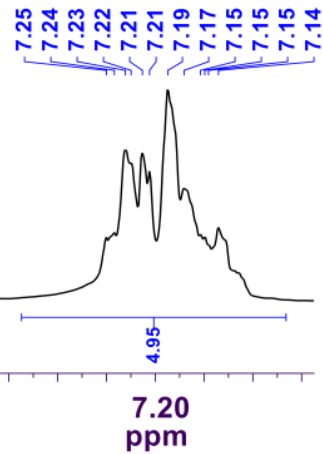

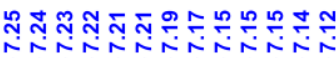

c

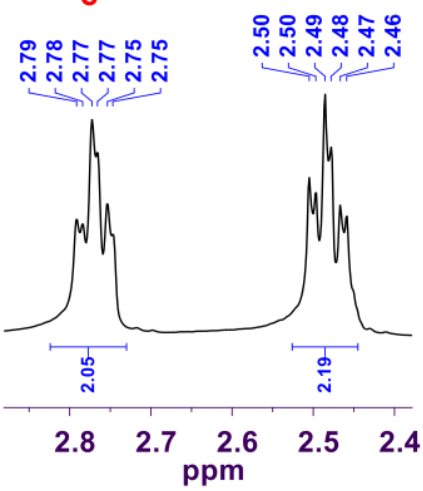

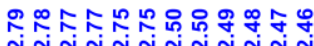

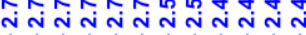

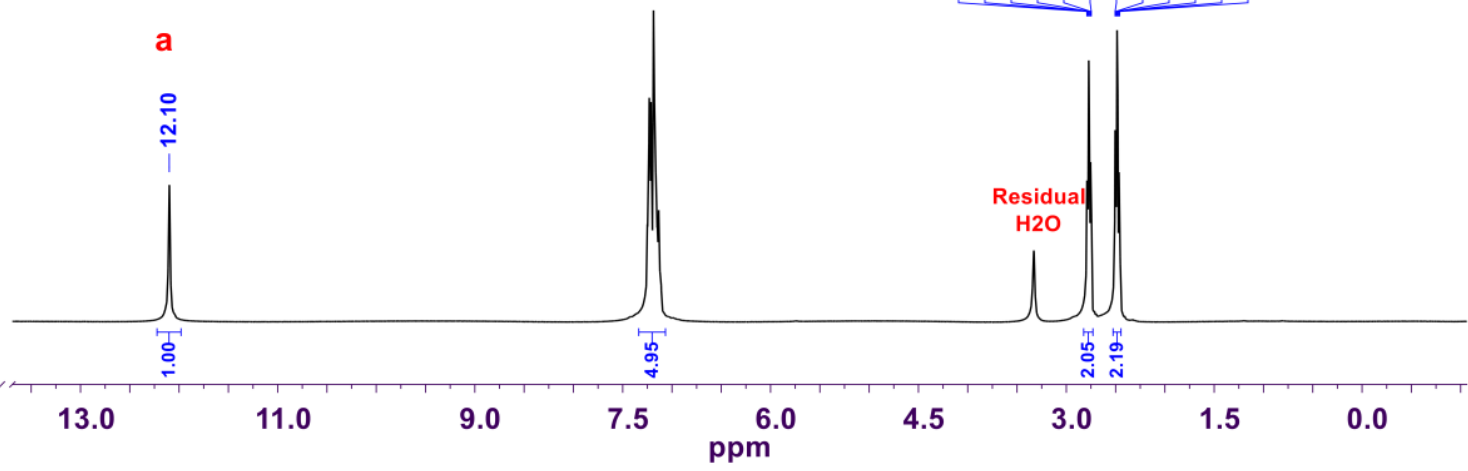

Figure S2. The ${ }^{1} \mathrm{H}-\mathrm{NMR}$ spectrum of the HCA synthesized by CTH reaction. 

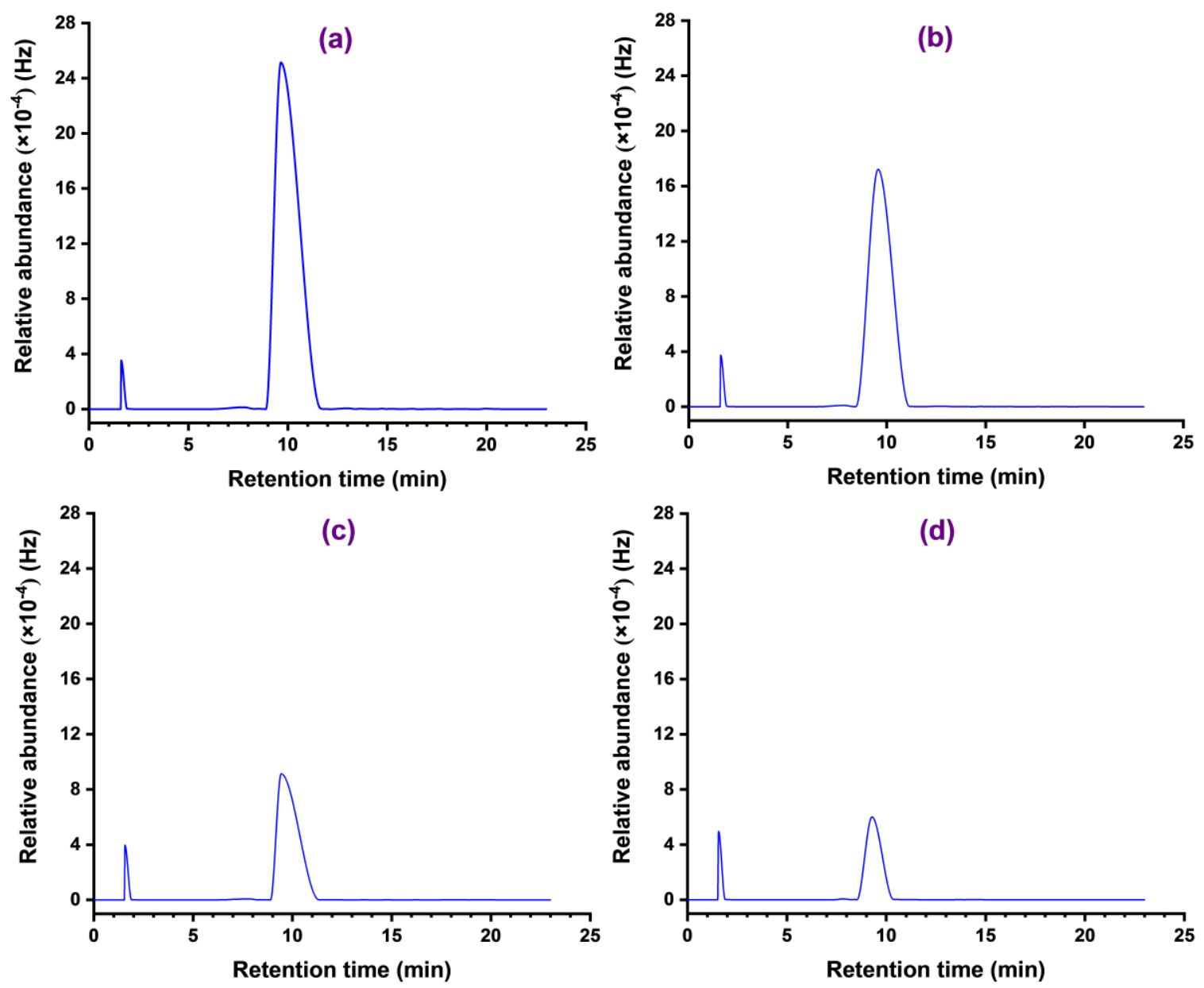

Figure S3. GC spectra for cinnamic acid (CA) standard solutions at the concentrations of (a) $4.05 \times 10^{-2}$, (b) $2.7 \times 10^{-2},(\mathrm{c})$ $1.35 \times 10^{-2}$, and (d) $6.75 \times 10^{-3}$ mol.L ${ }^{-1}$. 

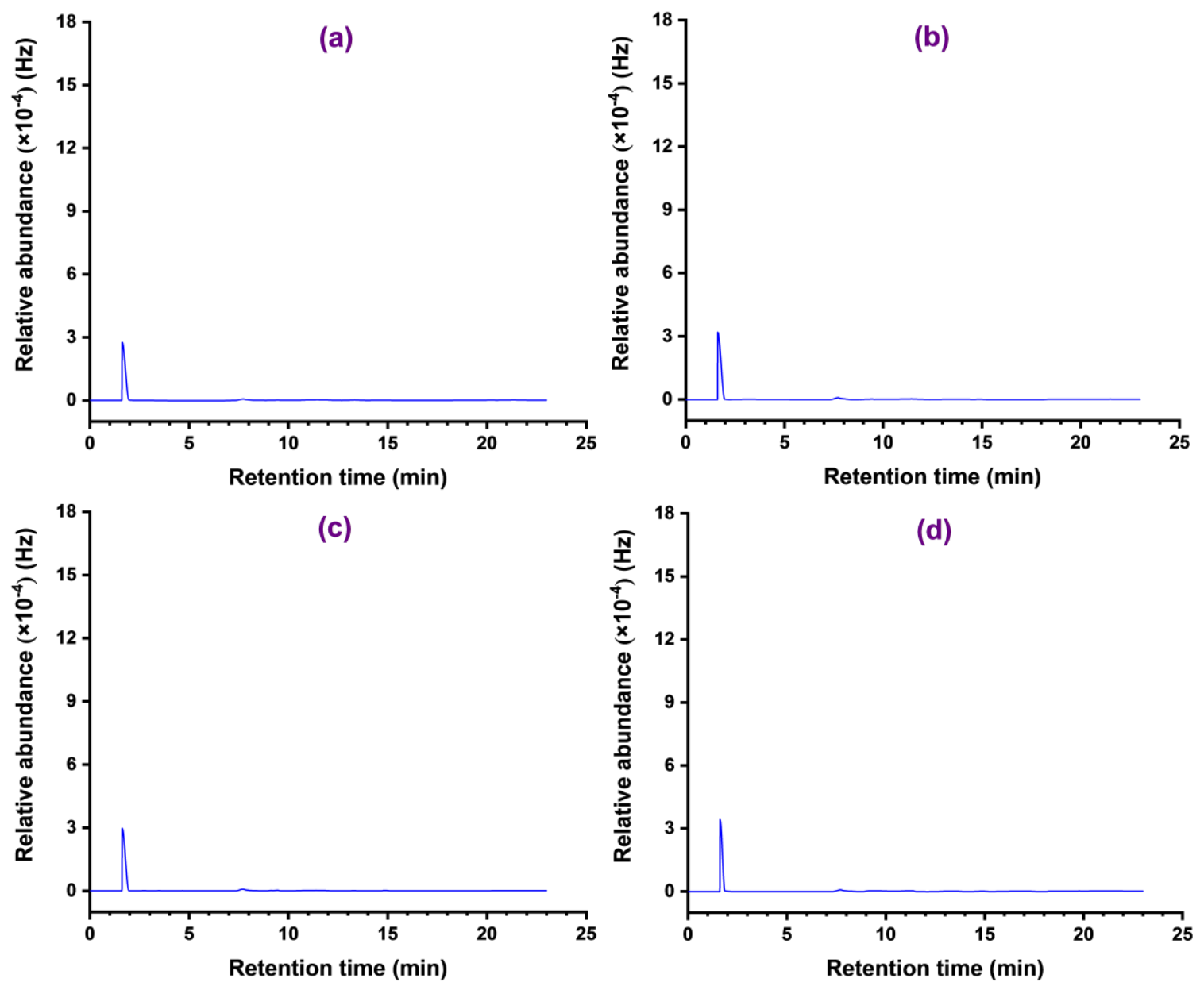

Figure S4. GC spectra for CTH reaction solutions in presence (a) $\mathrm{Pd} / \mathrm{AC}_{\mathrm{E}}$, (b) $\mathrm{Pd} / \mathrm{AC}_{\mathrm{A}},(\mathrm{c}) \mathrm{Pd} / \mathrm{AC}$, and (d) $\mathrm{Pd} / \mathrm{AC}$ catalysts at the $20 \mathrm{~min}$. 

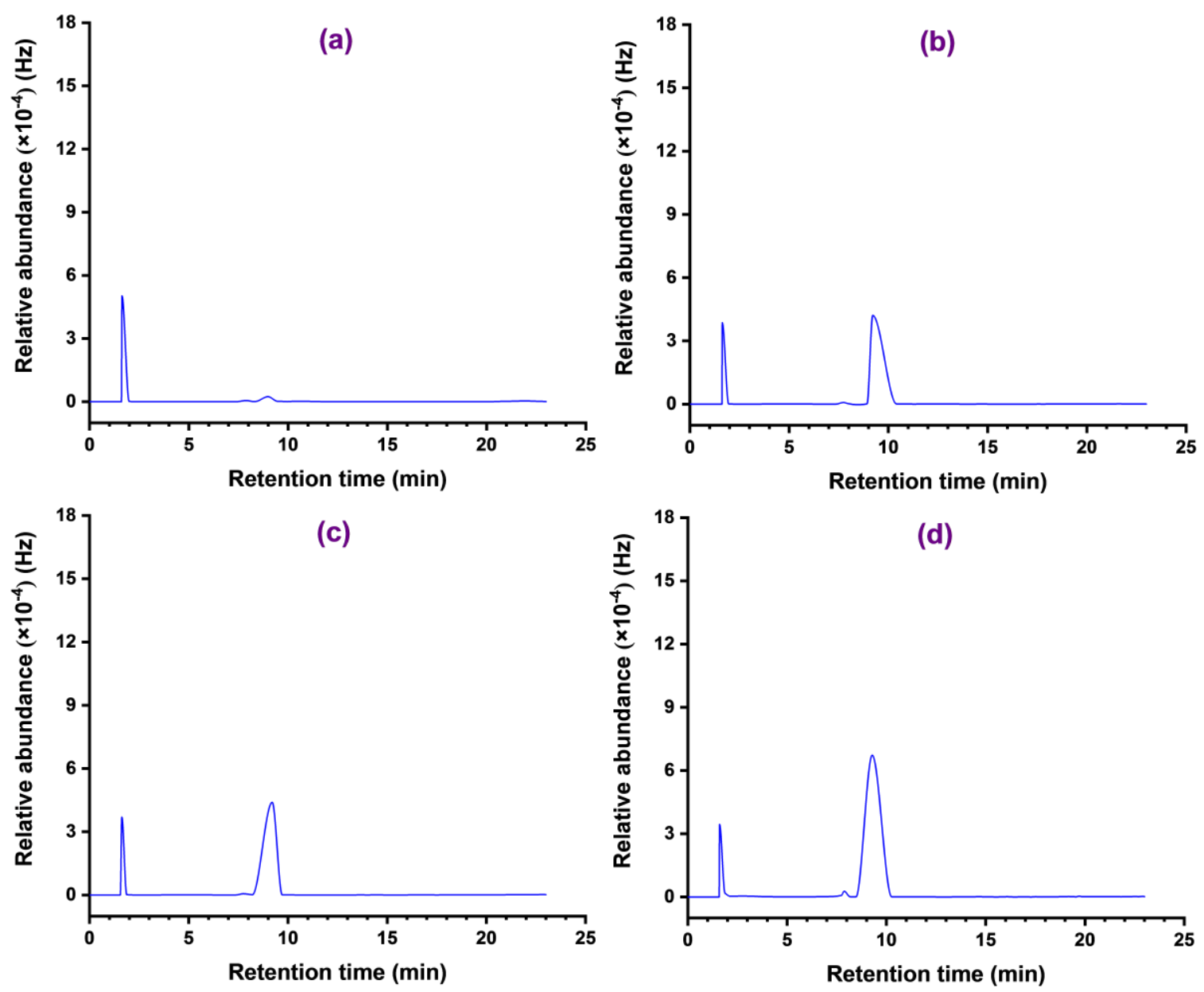

Figure S5. GC spectra for catalytic hydrogenation $(\mathrm{CH})$ reaction solutions in presence (a) $\mathrm{Pd} / \mathrm{AC}$, $\left(\right.$ b) $\mathrm{Pd} / \mathrm{AC} A,(c) \mathrm{Pd}_{\mathrm{AC}}$, and (d) $\mathrm{Pd} / \mathrm{AC}_{\mathrm{C}}$ catalysts at the $8 \mathrm{~min}$. 

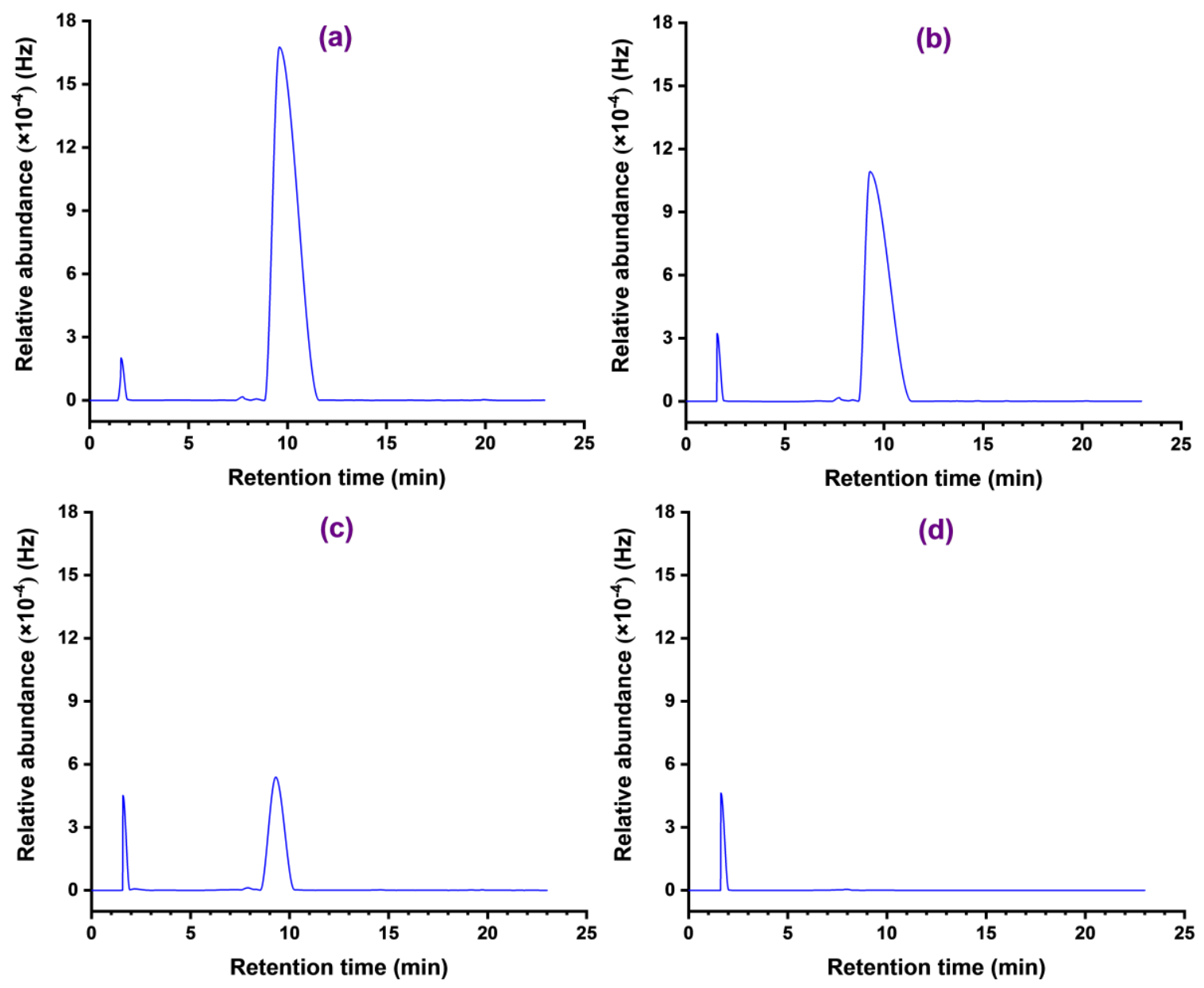

Figure S6. GC spectra for CTH reaction solutions in presence Pd/AC $\mathrm{E}$ catalyst at the (a) 5, (b) 10, (c) 15 , and (d) 20 minutes. 

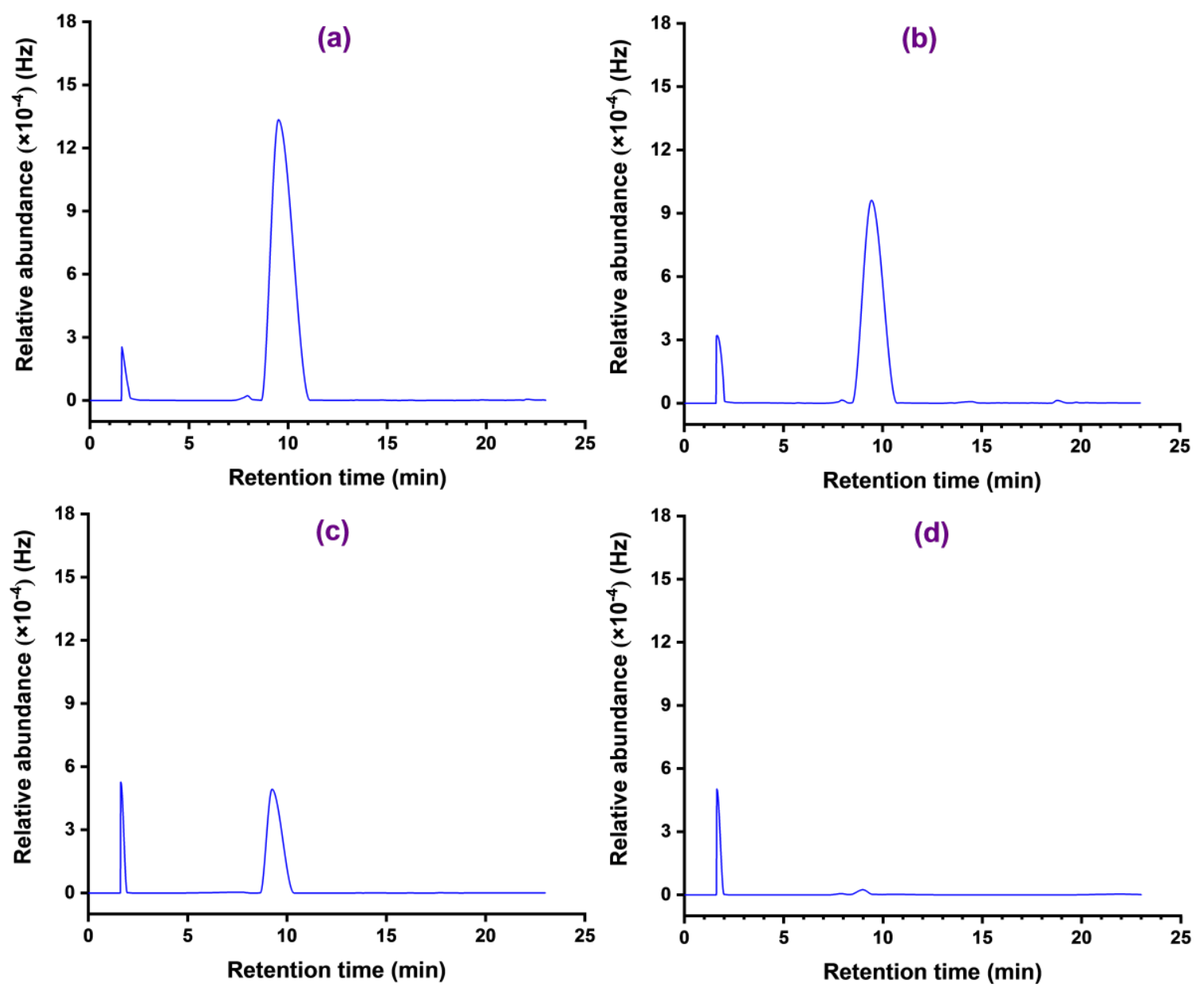

Figure S7. GC spectra for $\mathrm{CH}$ reaction solutions in presence $\mathrm{Pd} / \mathrm{AC}_{\mathrm{E}}$ catalyst at (a) 2, (b) 4, (c) 6, and (d) 8 minutes. 

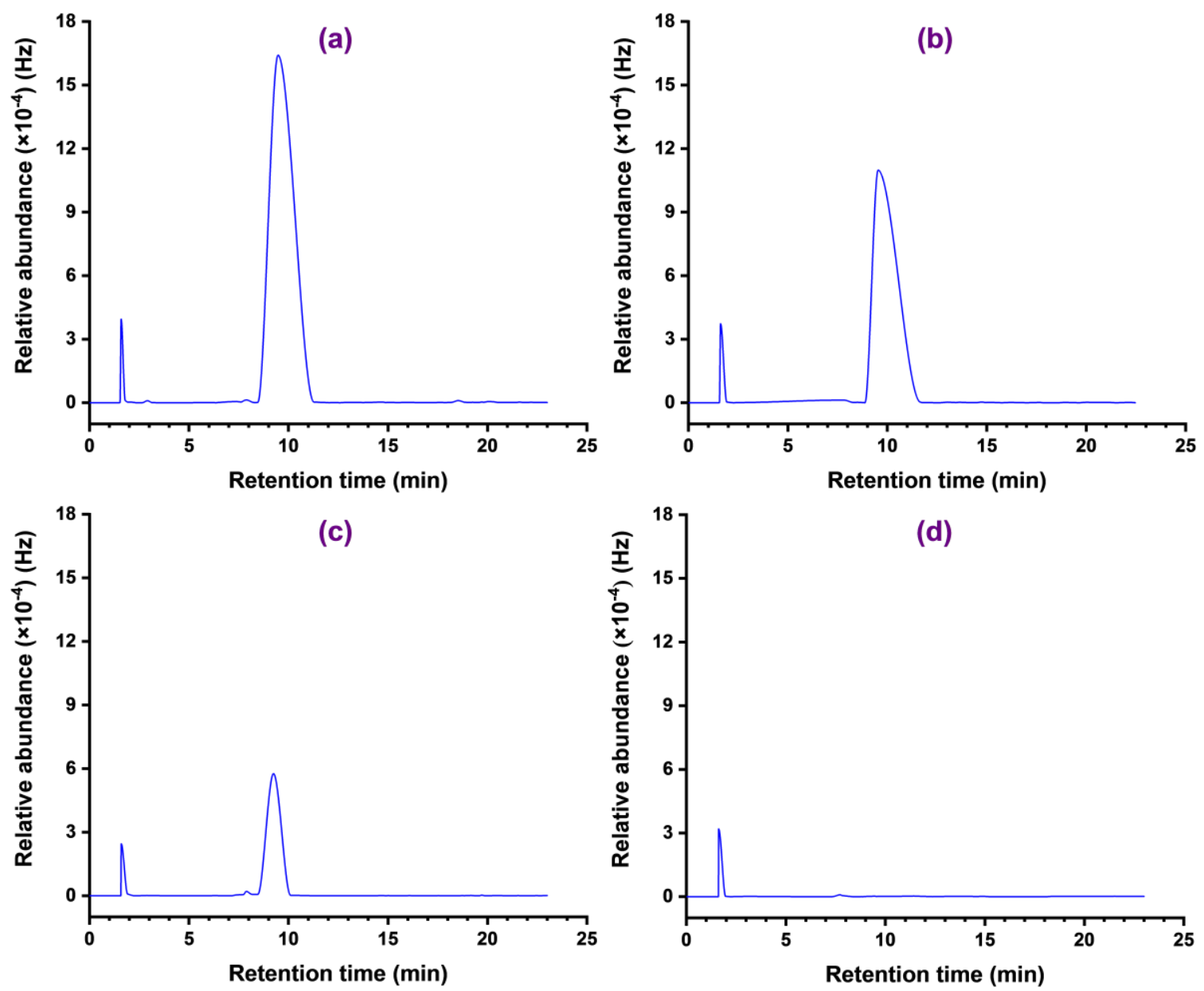

Figure S8. GC spectra for $\mathrm{CH}$ reaction solutions in presence $\mathrm{Pd} / \mathrm{AC}_{\mathrm{E}}$ catalyst at (a) 2, (b) 4, (c) 6, and (d) 8 minutes. 

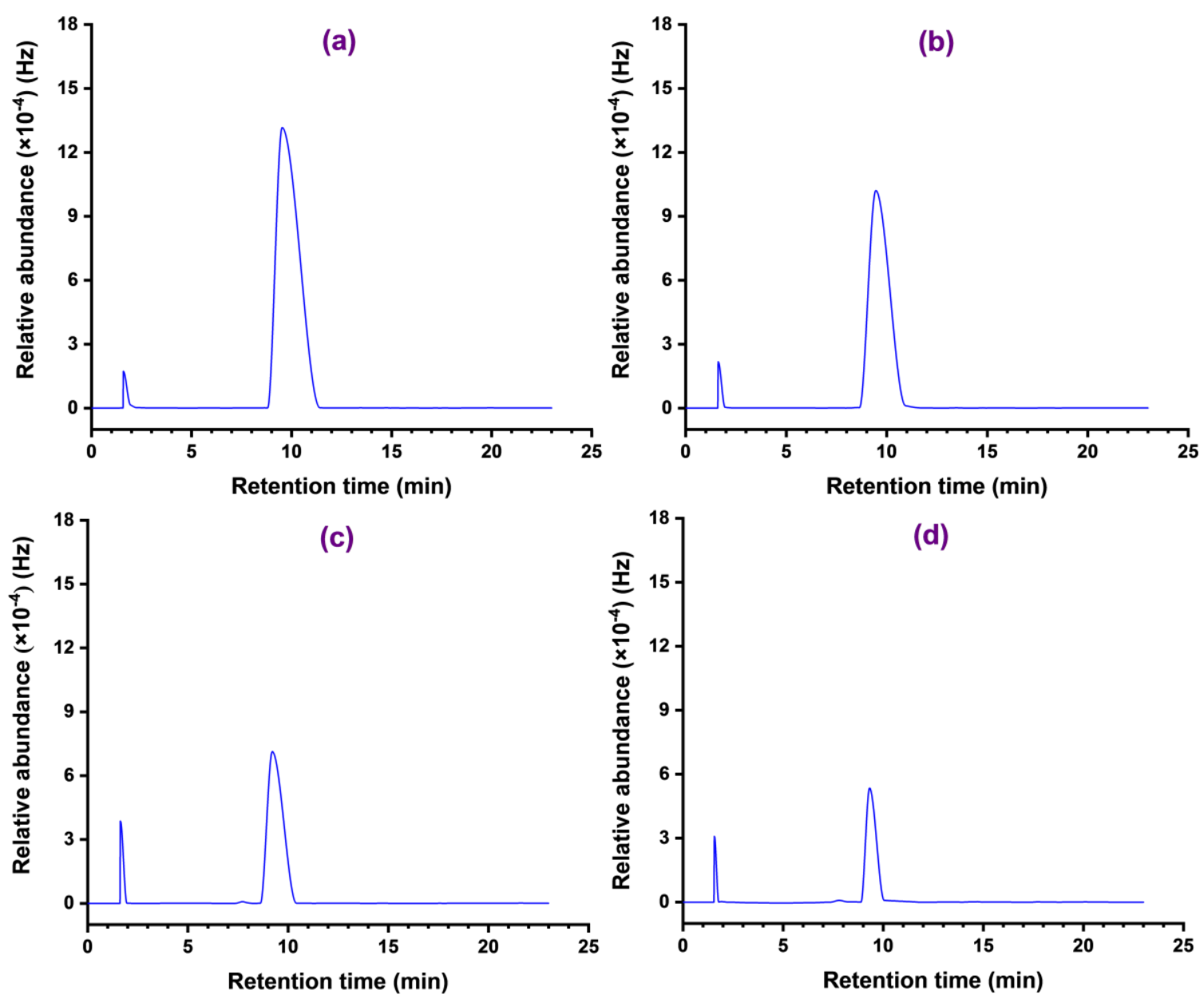

Figure S9. GC spectra for $\mathrm{CH}$ reaction solutions in presence $\mathrm{Pd} / \mathrm{AC}_{\mathrm{A}}$ catalyst at (a) 2, (b) 4, (c) 6, and (d) 8 minutes. 

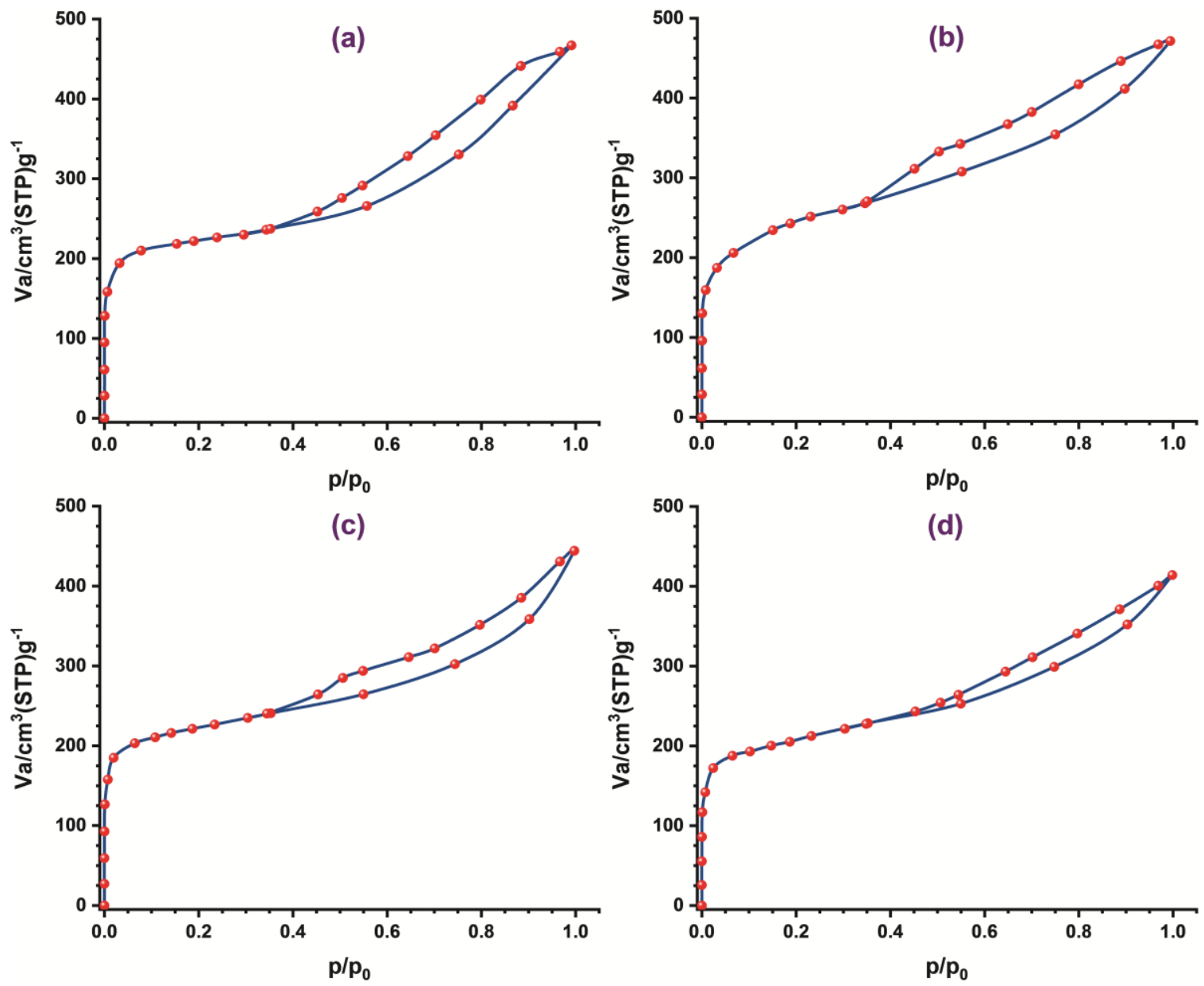

Figure S10. (A) Nitrogen adsorption-desorption isotherms for (a) $\mathrm{Pd} / \mathrm{AC}_{\mathrm{E}}$ (b) $\mathrm{Pd} / \mathrm{AC}_{\mathrm{A}}$ (c) $\mathrm{Pd} / \mathrm{AC} \mathrm{C}_{\mathrm{H}}$ and (d) $\mathrm{Pd} / \mathrm{AC} \mathrm{C}_{\mathrm{C}}$ catalysts. 


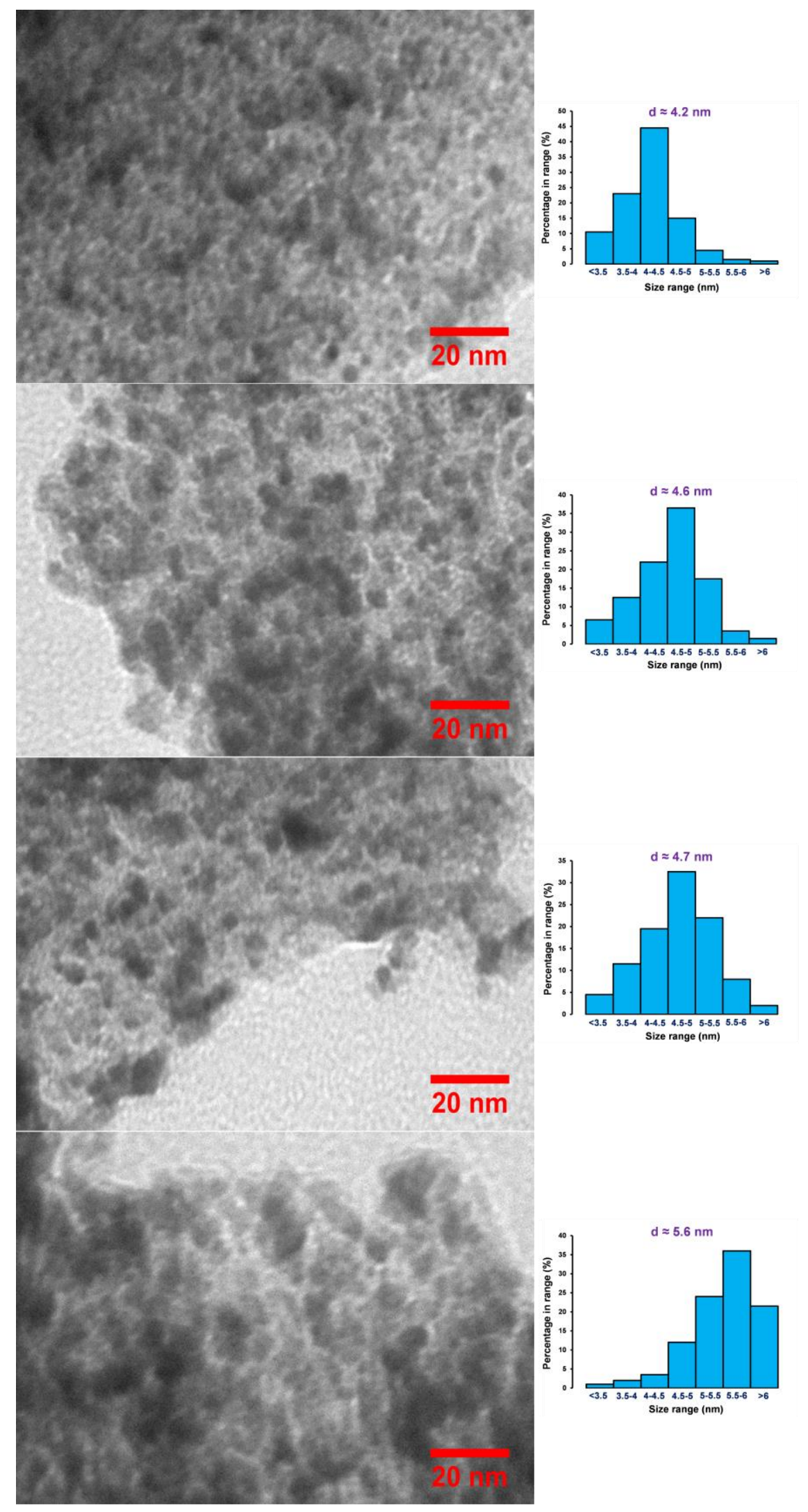

Figure S11. TEM images of (A) $\mathrm{Pd} / \mathrm{AC}_{\mathrm{E}}$, (B) $\mathrm{Pd} / \mathrm{AC}_{\mathrm{A}},(\mathrm{C}) \mathrm{Pd} / \mathrm{AC}_{\mathrm{H}}$, and (D) $\mathrm{Pd} / \mathrm{AC}_{\mathrm{C}}$ catalysts and histograms of their particle size distribution. 


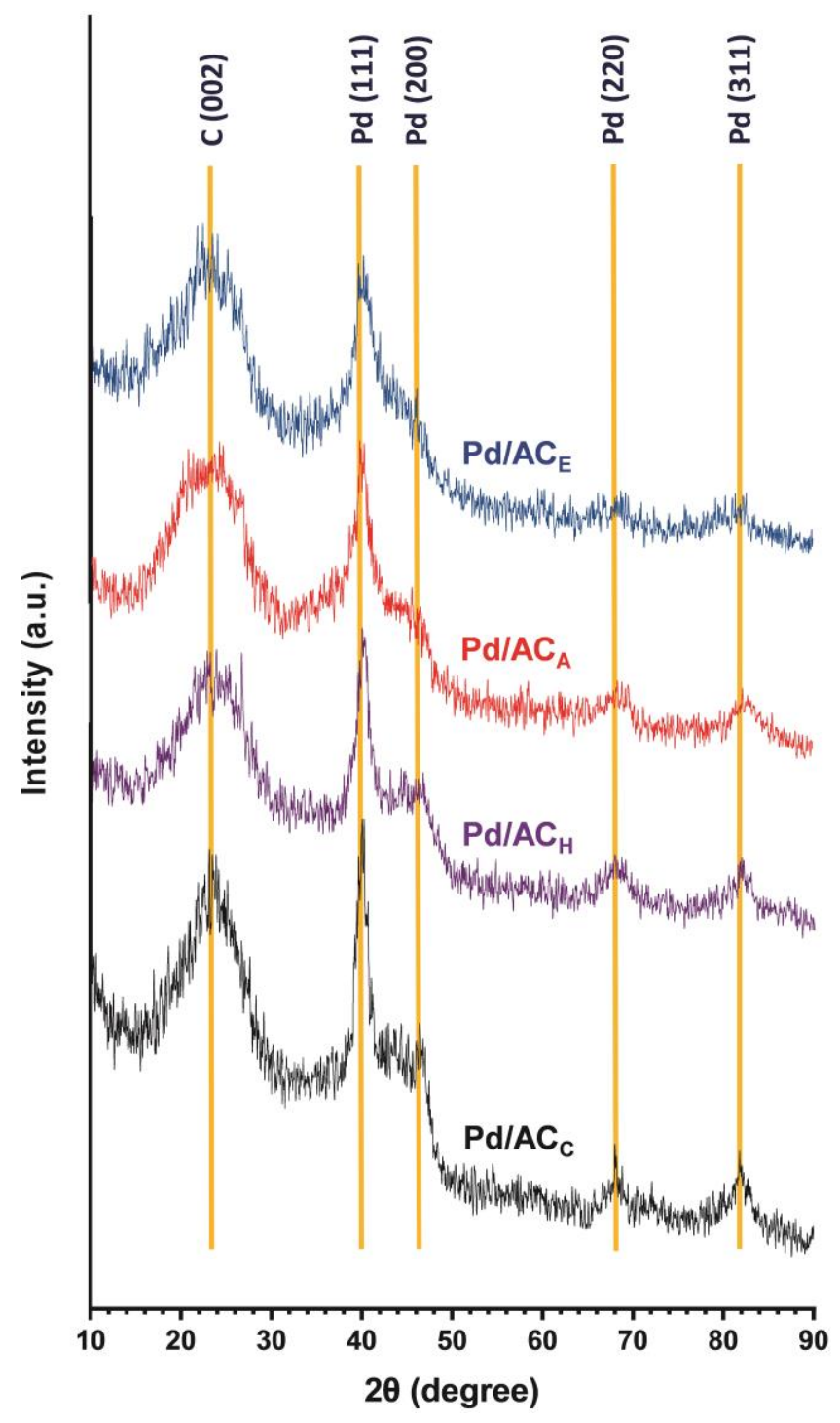

Figure S12. XRD patterns of $\mathrm{Pd} / \mathrm{AC}$ catalysts. 
Table S1. The results of the limit of detection (LOD) for the Bayer test in the CTH reaction.

\begin{tabular}{ccc}
\hline Weight ratio (CA:HCA) & Concentration of CA $\left(\mathrm{g} . \mathrm{L}^{-1}\right)$ & Observation \\
\hline$(0: 1)$ & 0 & purple color \\
$(0.005: 0.995)$ & 0.5 & purple color \\
$(0.01: 0.99)$ & 1 & brown suspension \\
$(0.02: 0.98)$ & 2 & brown suspension \\
\hline
\end{tabular}

${ }^{[a]}$ Weight ratio of $(0: 1)$ is blank solution HCA in tetralin

Table S2. The results obtained from the GC spectra for CA standard solutions.

\begin{tabular}{ccc}
\hline Standard & $\begin{array}{c}\text { CA Concentration in } \\
\text { standard solution }\left(\mathrm{mol}^{-1}\right)\end{array}$ & Peak area in GC \\
\hline 1 & 0 & 0 \\
2 & $6.7 \times 10^{-3}$ & $0.611 \times 10^{+6}$ \\
3 & $1.34 \times 10^{-2}$ & $1.231 \times 10^{+6}$ \\
4 & $2.68 \times 10^{-2}$ & $2.559 \times 10^{+6}$ \\
5 & $4.02 \times 10^{-2}$ & $3.825 \times 10^{+6}$ \\
\hline
\end{tabular}

Table S3. The results achieved by $\mathrm{GC}$ analysis for the $\mathrm{CTH}$ and $\mathrm{CH}$ reactions over $\mathrm{Pd} / \mathrm{AC}$ catalysts.

\begin{tabular}{|c|c|c|c|c|c|c|}
\hline Reaction & Catalyst & $\begin{array}{r}\text { Weight of } \\
\text { catalyst }(\mathrm{g}) \\
\end{array}$ & $\begin{array}{c}\text { Peak area in } \\
\text { GC }\end{array}$ & $\begin{array}{l}\text { CA concentration in } \\
\text { GC solution }\left(\mathrm{mol}^{\left.-L^{-1}\right)}\right.\end{array}$ & $\begin{array}{c}\text { Conversion } \\
\text { of CA }\end{array}$ & $\begin{array}{r}\text { Yield of } \\
\text { HCA }(\%)\end{array}$ \\
\hline \multirow{4}{*}{$\mathrm{CTH}$} & $\mathrm{Pd} / \mathrm{AC}_{\mathrm{E}}$ & $9 \times 10^{-3}$ & $0.66 \times 10^{+3}$ & $2.3 \times 10^{-4}$ & \multirow{4}{*}{0.991} & \multirow{4}{*}{98.7} \\
\hline & $\mathrm{Pd} / \mathrm{AC}_{\mathrm{A}}$ & $1.25 \times 10^{-2}$ & $1.048 \times 10^{+3}$ & $2.4 \times 10^{-4}$ & & \\
\hline & $\mathrm{Pd} / \mathrm{AC}_{\mathrm{H}}$ & $1.35 \times 10^{-2}$ & $0.668 \times 10^{+3}$ & $2.3 \times 10^{-4}$ & & \\
\hline & $\mathrm{Pd} / \mathrm{AC}_{\mathrm{C}}$ & $2 \times 10^{-2}$ & $1.166 \times 10^{+3}$ & $2.4 \times 10^{-4}$ & & \\
\hline \multirow{4}{*}{$\mathrm{CH}$} & $\mathrm{Pd} / \mathrm{AC}_{\mathrm{E}}$ & \multirow{4}{*}{0.1} & $0.152 \times 10^{+5}$ & $0.38 \times 10^{-3}$ & 0.986 & 98.2 \\
\hline & $\mathrm{Pd} / \mathrm{AC}_{\mathrm{A}}$ & & $3.459 \times 10^{+5}$ & $3.82 \times 10^{-3}$ & 0.857 & 85.4 \\
\hline & $\mathrm{Pd} / \mathrm{AC}_{\mathrm{H}}$ & & $3.661 \times 10^{+5}$ & $4.03 \times 10^{-3}$ & 0.849 & 84.6 \\
\hline & $\mathrm{Pd} / \mathrm{AC}_{\mathrm{C}}$ & & $6.673 \times 10^{+5}$ & $7.17 \times 10^{-3}$ & 0.732 & 73 \\
\hline
\end{tabular}


Table S4. The results obtained from GC spectra for $\mathrm{CTH}$ and $\mathrm{CH}$ reaction solutions in the presence $\mathrm{Pd} / \mathrm{AC} \mathrm{E}_{\mathrm{E}}$ catalyst at the different times of reaction.

\begin{tabular}{|c|c|c|c|c|c|}
\hline Reaction & $\begin{array}{l}\text { Reaction } \\
\text { time (min) }\end{array}$ & $\begin{array}{c}\text { Peak area in } \\
\text { GC }\end{array}$ & $\begin{array}{l}\text { CA concentration in } \\
\text { GC solution }\left(\mathrm{mol}^{\left.-\mathrm{L}^{-1}\right)}\right.\end{array}$ & $\begin{array}{l}\text { CA concentration in } \\
\text { reaction }{ }^{[\mathrm{a}]}\left(\mathrm{mol}^{\mathrm{L}} \mathrm{L}^{-1}\right)\end{array}$ & $\begin{array}{c}\text { Conversion } \\
\text { of CA } \\
\end{array}$ \\
\hline \multirow{4}{*}{$\mathrm{CTH}$} & 5 & $2.553 \times 10^{+6}$ & $2.677 \times 10^{-2}$ & 1.163 & 0 \\
\hline & 10 & $1.614 \times 10^{+6}$ & $1.702 \times 10^{-2}$ & 0.739 & 0.365 \\
\hline & 15 & $0.511 \times 10^{+6}$ & $0.554 \times 10^{-2}$ & 0.241 & 0.793 \\
\hline & 20 & $0.66 \times 10^{+3}$ & $0.233 \times 10^{-3}$ & $0.101 \times 10^{-1}$ & 0.991 \\
\hline \multirow{4}{*}{$\mathrm{CH}$} & 2 & $1.806 \times 10^{+6}$ & $1.901 \times 10^{-2}$ & 0.374 & 0.29 \\
\hline & 4 & $1.197 \times 10^{+6}$ & $1.267 \times 10^{-2}$ & 0.249 & 0.527 \\
\hline & 6 & $0.461 \times 10^{+6}$ & $0.502 \times 10^{-2}$ & $0.989 \times 10^{-1}$ & 0.812 \\
\hline & 8 & $0.152 \times 10^{+5}$ & $0.384 \times 10^{-3}$ & $0.756 \times 10^{-2}$ & 0.986 \\
\hline
\end{tabular}

${ }^{[a]}$ Initial concentration of CA in $\mathrm{CTH}$ and $\mathrm{CH}$ reactions are 1.164 and 0.527 moL.L ${ }^{-1}$, respectively.

Table S5. The results of GC spectra for $\mathrm{CTH}$ and $\mathrm{CH}$ reaction solutions over the $\mathrm{Pd} / \mathrm{AC}_{\mathrm{A}}$ catalyst at different times of reaction.

\begin{tabular}{|c|c|c|c|c|c|}
\hline Reaction & $\begin{array}{l}\text { Reaction } \\
\text { time (min) }\end{array}$ & $\begin{array}{c}\text { Peak area in } \\
\text { GC }\end{array}$ & $\begin{array}{l}\text { CA concentration in } \\
\text { GC solution }\left(\text { mol. }^{-1}\right)\end{array}$ & $\begin{array}{l}\text { CA concentration in } \\
\text { reaction }{ }^{[a]}\left(\text { mol.L }^{-1}\right)\end{array}$ & $\begin{array}{c}\text { Conversion } \\
\text { of CA }\end{array}$ \\
\hline \multirow{4}{*}{$\mathrm{CTH}$} & 5 & $2.556 \times 10^{+6}$ & $2.681 \times 10^{-2}$ & 1.164 & 0 \\
\hline & 10 & $1.685 \times 10^{+6}$ & $1.775 \times 10^{-2}$ & 0.771 & 0.337 \\
\hline & 15 & $0.528 \times 10^{+6}$ & $0.571 \times 10^{-2}$ & 0.248 & 0.787 \\
\hline & 20 & $1.048 \times 10^{+3}$ & $0.237 \times 10^{-3}$ & $0.103 \times 10^{-1}$ & 0.991 \\
\hline \multirow{4}{*}{$\mathrm{CH}$} & 2 & $1.902 \times 10^{+6}$ & $2.001 \times 10^{-2}$ & 0.394 & 0.253 \\
\hline & 4 & $1.302 \times 10^{+6}$ & $1.377 \times 10^{-2}$ & 0.271 & 0.486 \\
\hline & 6 & $0.705 \times 10^{+6}$ & $0.756 \times 10^{-2}$ & 0.149 & 0.718 \\
\hline & 8 & $0.346 \times 10^{+6}$ & $0.382 \times 10^{-2}$ & $0.753 \times 10^{-1}$ & 0.857 \\
\hline
\end{tabular}

${ }^{[\mathrm{a}]}$ Initial concentration of CA in $\mathrm{CTH}$ and $\mathrm{CH}$ reactions are 1.164 and 0.527 moL. $\mathrm{L}^{-1}$, respectively. 